\title{
A rodent model of anterior ischemic optic neuropathy (AION) based on laser photoactivation of verteporfin
}

\author{
Jing-yu Min ${ }^{1,2,3}$, Yanan Lv ${ }^{1,2,3}$, Lei Mao ${ }^{1,2,3}$, Yuan-yuan Gong ${ }^{1,2,3^{*}} \mathbb{D}$, Qing Gu ${ }^{1,2,3}$ and Fang Wei ${ }^{1,2,3}$
}

\begin{abstract}
Background: A rodent model of photodynamic AION resulting from intravenous verteporfin is presented. The analysis of the morphological function, the pathological changes and the potential mechanism of action were further investigated.

Methods: Photodynamic treatment was conducted on the optic nerve head (ONH) following administration of the photosensitizer. The fellow eye was considered as sham control. Fundus Fluorescein angiography (FFA), spectral domain optical coherence tomography (SD-OCT) and Flash-visual evoked potential (F-VEP) recordings were conducted at different time points. Immunohistochemistry was used to observe apoptotic cell death (TUNEL) and macrophage infiltration (ED-1/lba-1). Retrograde labeling of retinal ganglion cells (RGCs) was used to evaluate the loss of RGCs.

Results: After laser treatment, SD-OCT indicated optic nerve edema, while FFA indicated late leakage of the ONH. F-VEPs were distinctly reduced compared to control eyes. The number of apoptotic RGCs peaked on day 14 $(5.71 \pm 0.76, p<0.01)$. The infiltration of ED-1 and Iba-1 increased on the 3rd day following PDT, while it peaked on day 14 (67.5 \pm 9.57 and $77.5 \pm 12.58$ respectively, $p<0.01$ ). Following 3 weeks of AION, the densities of RGCs in the central retinas of the normal and AION eyes were $3075 \pm 298 / \mathrm{mm}^{2}$ and $2078 \pm 141 / \mathrm{mm}^{2}(p<0.01)$, respectively.

Conclusions: Verteporfin photodynamic treatment on rodents $\mathrm{ONH}$ can lead to functional, histological, and pathological changes. This type of animal model of AION is easy to establish and stable. It can be used for studying the mechanism and neuroprotective medicine of AION injury.
\end{abstract}

Keywords: Anterior ischemic optic neuropathy, Verteporfin, Laser photoactivation, RGCs

\section{Introduction}

Anterior ischemic optic neuropathy (AION) is the leading cause of sudden optic nerve-related (ON-related) vision loss in elderly people. Approximately $11.7 \%$ of these cases experience central vision abnormalities [1]. In addition, AION was notably noted in one eye, according to a previous study conducted in China, with an incidence rate of $0.03+/-0.03 \%$ (mean $+/$ - standard error) per 5 years (1:16,000 subjects annually) [2].

\footnotetext{
* Correspondence: gyydr@alumni.sjtu.edu.cn

'Department of Ophthalmology, Shanghai General Hospital, Shanghai Jiao Tong University School of Medicine, NO.100, Haining Road, Hongkou District, Shanghai 200080, China

${ }^{2}$ Shanghai Key Laboratory of Ocular Fundus Diseases, NO.100, Haining Road, Hongkou District, Shanghai 200080, China

Full list of author information is available at the end of the article
}

Based on clinical studies, AION is considered a secondary symptom to ischemia, which is predominantly caused by the posterior ciliary arteries [3], although the exact pathophysiology that leads to axonal degeneration remains undiscovered. In addition, there are a lot of risks, scuh as diabetes mellitus, hypertension, hypercholesterolemia, and crowded structure of the optic disc with small cup to disc ratio. To date no effective method has been reported to prevent vision loss following the development of AION. Thus, it is necessary to establish an animal model of AION that can be used to assess the potential benefits of neuroprotective strategies.

Bernstein et al. conducted the first study that used a photodynamic model of AION in rats by photoactivating rose Bengal with a $532 \mathrm{~nm}$ laser [4]. Since then, this 
model had been further investigated by several neuroprotective agents [5-8]. Differences had been noted among the severity of this disease in rodents due to a variety of factors, such as the differences of operation and individuals worked, and the very short half-life of rose Bengal, which required a rapid operation of photoactivation following administration. Furthermore, the photosensitizing agent mesoporphyrin IX dihydrochloride was used in establishing the model of AION via intraperitoneal injection [9], although this type of model had not been widely used due to its short time of discovery.

Verteporfin is a benzoporphyrin derivative and is proposed for treating several eye diseases, such as choroidal neovascularization (CNV), pathological myopia, and/or polypoidal choroidal vasculopathy (PCV). Photodynamic treatment following intravenous injection of verteporfin in rodents can lead to edema of the normal choroid and retina [10]. Hence this type of photosensitizer was used in the development of the AION model. Furthermore, verteporfin exhibits a longer half-life than rose Bengal, whereas the photosensitizing agents that were used in the present study were recycled by the discarded parts used for the clinical procedures required for AION examination. Consequently, verteporfin was selected to construct the AION model in the current study.

In the present study, we describe an alternative approach to induce AION inrodents. Furthermore, we studied the morphology, function, and the mechanism of action of this model.

\section{Method}

\section{Animals}

All animal experiments adhered to the ARVO statement for the Use of Animals in Ophthalmic and Vision Research. Adult male Sprague Dawley (S-D) rats weighing 180-200 g were purchased from the Shanghai Laboratory Animal Center of the Chinese Academy of Sciences and were used for all the experiments. All animals were housed in cages at constant temperature, fed with a standard diet ad libitum and maintained under a 12-h light/12-h dark photoperiod. All types of surgery and manipulation were conducted in the Shanghai Key Laboratory of Fundus Disease. All procedures were carried out under sedation. Sedation was achieved by intraperitoneal injection of $10 \%(w / v)$ chloral hydrate $(3.5 \mathrm{ml} / \mathrm{kg})$. The pupils of anesthetized rats were dilated with one drop of $5 \%$ tropicamide, and the corneal was anesthetized with one drop of $0.4 \%$ oxybuprocaine hydrochloride. The number of animals used for the morphological and functional analyses of each group was $N=6$.

\section{Induction of AION}

A total of $6 \mathrm{mg} / \mathrm{m}^{2}$ of verteporfin (Novartis Ophthalmics Europe Ltd., Basel, Switzerland) was injected intravenously through the tail vein in order to induce optic nerve head ischemia. Following administration of the photosensitizer for 1 to $10 \mathrm{~min}$ duration, the laser was applied on the $\mathrm{ONH}$ of the left eye of each animal. The optic nerve head was subjected to a laser beam at a wavelength of $689 \mathrm{~nm}$ across a $500-\mu \mathrm{m}$ diameter spot size for 158 consecutive secs. The laser energy used was $600 \mathrm{~mW} / \mathrm{cm}^{2}$. The laser beam was used for the fellow eye at the $\mathrm{ONH}$ with no laser emission and the same operation parameters as stated above.

\section{Fluorescein angiography and optical coherence tomography}

Fundus fluorescein angiography (FFA), and spectral domain optical coherence tomography (SD-OCT) were conducted on days $0,1,3$ and 7 following treatment to observe the progress of the optic nerve head edema and the related retinal response. A solution of $10 \%$ Fluorescein sodium (Alcon Laboratories Inc. Switzerland) was injected intraperitoneally. The angiographs were recorded following the change in coloration of the conjunctiva that appeared yellow in color (Heidelberg Engineering, Heidelberg,Germany). Retinal structure and retinal thickness were measured using SD-OCT (Heidelberg Engineering). Retinal scans were centered on the optic disc in both control and injured eyes.

\section{Flash-visual evoked potentials (F-VEPs)}

The F-VEP was conducted on days 1, 7, 14 and 21 following treatment with a Ganzfeld system (RetiPort, Roland Consult, Brandenburg, Germany). All animals had their pupils dilated and were anesthetized. Upon examination of one of the two eyes of each animal, the contralateral eye was covered. The settings of F-VEPs were based on previous reports $[6,11]$, including no background illumination, a flash intensity of Ganzfeld $0 \mathrm{~dB}$, a single flash with a flash rate of $1.9 \mathrm{~Hz}$ and a flash intensity of $3 \mathrm{~cd} . \mathrm{s} / \mathrm{m}^{2}$. The average test was conducted at 80 sweeps, whereas the threshold for rejecting artifacts was set at $50 \mathrm{mV}$ and a sample rate of $2,000 \mathrm{~Hz}$ was used. The amplitudes of P1 for each F-VEP wave within the initial 100-ms interval were determined and used for the amplitude analysis (amplitude of $\mathrm{P}_{1}=$ amplitude of $\mathrm{P}_{1}$ - amplitude of $\mathrm{N}_{2}$ ) $[6,12]$.

\section{Immunohistochemistry}

Animals were euthanized on days 1, 4, 7, 14 and 21 following laser application. The eyes were enucleated, fixed in $4 \%$ paraformaldehyde (PFA) in PBS for $24 \mathrm{~h}$ and the anterior segment was removed. Subsequently, certain eyes were dehydrated in $30 \%$ sucrose overnight and embedded in Tissue-Tek O.C.T compound (Sakura, Torrance, CA) Cross sections of $10-\mu \mathrm{m}$ in diameter were performed. The sections were incubated with mouse 
anti-CD68 monoclonal antibody(Serotec Ltd., Oxford, UK) at a 1:100 dilution and/or rabbit anti-Iba-1 monoclonal antibody (Abcam Inc. Cambridge, MA) at a 1:100 dilution, at $4{ }^{\circ} \mathrm{C}$ overnight in order to identify macrophages and microglia. FITC conjugated goat anti-mouse IgG and/or FITC conjugated goat anti-rabbit IgG (Jackson Immunoresearch, West Point, PA) were incubated with the sections for $1 \mathrm{~h}$. Finally, sections were counterstained with 4',6-diamidino-2-phenylindole (DAPI) nuclear stain.

The remaining eyes that were not examined were dehydrated with a posterior eyecup and embedded in paraffin. Retinal cross sections (5 mm thick) were then cut and stained with hematoxylin and eosin (Sigma, MO,USA). The sections were photographed and measured at approximately 2 to 3 disc diameters from the optic nerve using a microscope (Olympus BX53; Olympus, Tokyo, Japan). The thickness of the retinal tissues was determined by cell counts over a distance scale of $200 \mathrm{~mm}$. The retinal thickness and cell number were calculated as the mean values of at least 3 measurements in adjacent sections [13].

\section{TdT-mediated dUTP Nick-end labeling (TUNEL) assay} On days 1, 4, 7 and/or 14 following laser application, paraffin-embedded retinal tissue sections were deparaffinized, rehydrated, fixed with $4 \%$ PFA for $15 \mathrm{~min}$ at $4{ }^{\circ} \mathrm{C}$ and then subjected to enzymatic digestion with $20 \mathrm{mg} / \mathrm{ml}$ proteinase $\mathrm{K}$ for 8 to $10 \mathrm{~min}$ at room temperature. Induction of apoptosis was examined by TUNEL assay using a DeadEnd $^{\text {mit }}$ Fluorometric TUNEL System, according to the manufacturer's instructions. 4',6-diamidino-2-phenylindole (DAPI) was used to stain the nuclear regions of the tissues. TUNEL-positive cells were examined using a laser scanning confocal microscope (Zeiss LSM 510, Carl Zeiss,Germany) in vitro in 6 random fields (at least 100 DAPI-positive cells per field) for each experimental group. The level of apoptosis was expressed as the ratio of the number of TUNELpositive cells to that of DAPI-positive cells [13].

\section{Retrograde labeling of RGCs with FluoroGold and morphometry of the RGCs}

Following deep anesthesia, the rat heads were fixed in a stereotactic apparatus (Stoelting Kiel, Germany) and the skin covering the skull of the rats was incised. Fluoro-Gold (FG;Biotium, Hayward, CA, USA) was injected ( $2 \mu \mathrm{l}$ of $4 \%$ FG in distilled $\mathrm{H}_{2} \mathrm{O}$ ) into the superior colliculus (SC) on each side using a microsyringe, and was retained for $10 \mathrm{~min}$. The animals were maintained for 1 week post-labeling and subsequently the eyes were enucleated and fixed with 4\% PFA for $1 \mathrm{~h}$. The retinas were examined with an Olympus BX53 fluorescence microscope (Olympus, Tokyo, Japan) with UV excitation (excitation filter, 350-400 nm; barrier filter, $515 \mathrm{~nm}$ ) and a digital imaging system. The RGCs were examined by division into 4 quadrants (superior, inferior, nasal,and temporal), which were further divided into central $(0.8-1.2 \mathrm{~mm}$ from the optic disc), middle $(1.8-2.2 \mathrm{~mm}$ from the optic disc), and peripheral regions $(0.8-1.2 \mathrm{~mm}$ from the retinal border). A total of 2 standard square areas $\left(200 \times 200 \mu^{2}\right)$ were measured in each region. The density of RGCs in each group of rats was expressed as the number of labeled RGCs $/ \mathrm{mm}^{2}$ compared with the counted retinal area [14].

\section{Statistical analysis}

The quantitative data were presented as mean $\pm \mathrm{SD}$. Statistical analyses were conducted using SPSS version 20 (SPSS, IL, USA). An unpaired Student's t-test for two-group data and one-way analysis of variance followed by a post hoc Bonferroni's multiple comparison test for three groups or more. A $p$ value of lower than 0.05 $(p<0.05)$ was considered statistically significant. Each experiment was conducted three times.

\section{Results \\ Morphology of ONH following AION induction}

The control eye had no abnormal changes (Fig. 1a, d). In the photodynamically treated eyes, FFA indicated fluorescein leakage from the $\mathrm{ONH}$ vasculature on day 1 , (Fig. 1b, c) which was consistent with the swelling of the retina tissue as demonstrated by the SD-OCT images. On day 3, the optic nerve edema was more pronounced (Fig. 1e). However, a resolution of the edema was noted on day 7.

\section{Flash-visual evoked potentials (F-VEPs)}

F-VEPs were recorded at the first, second and third week, following AION induction in order to evaluate the function of ON (Fig. 2). The F-VEPs of the treated eyes were compared with the fellow eyes of each animal in order to eliminate the variations in the F-VEP amplitude within the animal groups [15]. F-VEP amplitudes in the treated eyes $(N=6)$ were estimated to $87.3 \pm 11 \%, 67.6 \pm$ $11.5 \%$ and $35.9 \pm 13.6 \%$ of the fellow control eyes at the first, second and third week, respectively $(P<0.05)$. F-VEP latencies in the two data sets exhibited no statistical significance, as demonstrated in previous experiments [15].

\section{Induction of apoptosis and death in the RGC layer}

The induction of apoptosis was evident by the presence of TUNEL-positive cells in the outer nuclear layer (ONL), inner nuclear layer (INL) and RGC layers of the control eyes. The present study analyzed solely the induction of apoptosis in the RGC layer in treated and sham eyes. On the 1st and the 3rd day following AION induction, negligible induction of apoptosis was noted by the presence of TUNEL- positive cells in GCL, which were not demonstrated in the corresponding figure. On 


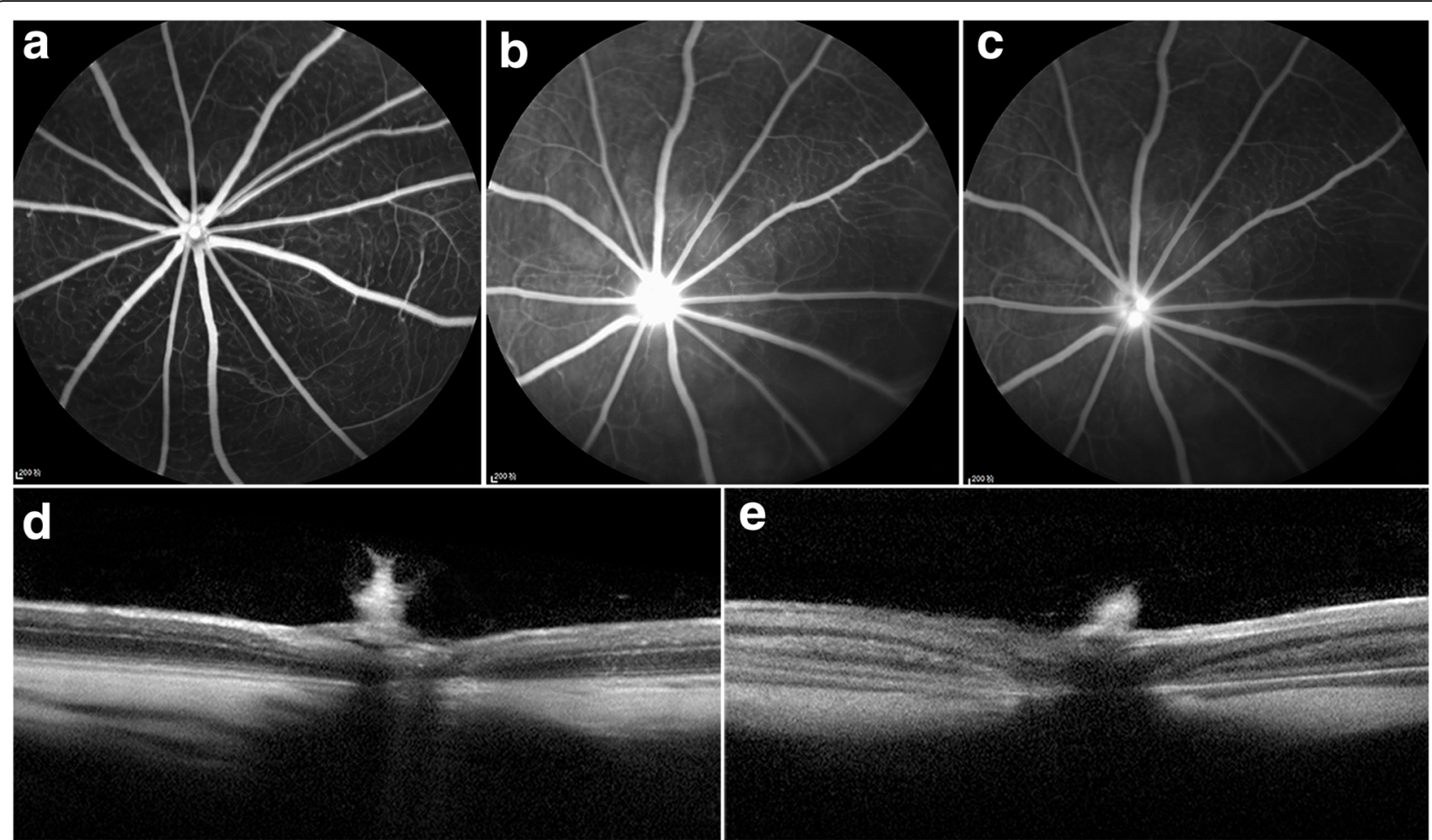

Fig. 1 Fundus fluorescein angiography (FFA) and spectral domain optical coherence tomography (SD-OCT). a, d The control fellow eye had no abnormal changes. $\mathbf{b}$ In the mid-phase, hyperfluorescence can be dectected at the ONH in PDT eye at 1 day post-AION. c In the late-phase, subdued fluorescence at ONH in the same PDT eye. e SD-OCT showed the swelling of the retina tissue of treated eyes at 3 days post-AION

day 7 , the number of TUNEL-positive cells significantly increased, and reached a peak $(5.71 \pm 0.76, p<0.01)$ by day 14 , (Fig. 3b, d) then reduced. At 3 weeks post-AION, the densities of RGCs in the central retinas were 3,075 \pm $298 / \mathrm{mm}^{2}$ and 2,078 $\pm 141 / \mathrm{mm}^{2}$ in the normal and AION eyes, respectively, while in the mid-peripheral retinas the corresponding densities were $2,615 \pm 138 / \mathrm{mm}^{2}$ and $1,691 \pm 142 / \mathrm{mm}^{2}$, respectively (Fig. 3a, c). The densities of RGCs exhibited significant variation in the treated group compared with the sham group $(N=6$ in each group, all $p<0.01$ ).

\section{The inflammatory response in retina and $\mathrm{ON}$}

In control ONs, occasional ED-1(+) cells were noted in the peripapillary choroid and in the anterior ON surrounding a blood vessel. By contrast, distributed Iba-1(+) cells were noted in the $\mathrm{ON}$, in the peripapillary choroid, and around the blood vessels (Fig. 4). Following 3 days of treatment, scattered ED-1(+) cells exhibited a moderate increase notably in the choroid and in close proximity to the blood vessels compared with the normal eyes, while Iba-1(+) cells exhibited a significant localization on the anterior ON. On day 14, ED(+) and Iba-1(+) cells were widely distributed on $\mathrm{ON}$, and both reached their maximum number $(67.5 \pm 9.57$ and $77.5 \pm 12.58$ respectively, $p<0.01)$ during the whole process of the study. Subsequently, ED-1 cells tended to disappear, whereas Iba-1(+) cells exhibited a considerable decrease to a certain number of cells.

\section{Discussion}

In the present study, we aimed to establish an alternative experimental model of laser photoactivation that can be applied for the development of neuroprotective agents against AION. Bernstein et al. were the first to develop a photodynamic model of AION in rats by photoactivating rose Bengal with a $532 \mathrm{~nm}$ laser [4]. Since then, this model had been further utilized by various research groups [15-18]. However, the biological half-life of rose Bengal in rats is solely 2 min [19], which indicates that the delay of operation post-injection may weaken the effect of photodynamic treatment on the ON. In the present study, the immediate operation with laser on $\mathrm{ON}$ was conducted following injection that can be technically challenging and can lead to variable injured levels of ON. Depending on our clinical experience and previous studies $[20,21]$, we selected an alternative treatment to induce AION using a dye with easier acquisition and longer half-life.

Preliminary experiments were conducted in order to simplify the experimental operation. Verteporfin was injected via intraperitoneal rather than intravenous injection, but it proved unsuccessful despite the high dose 

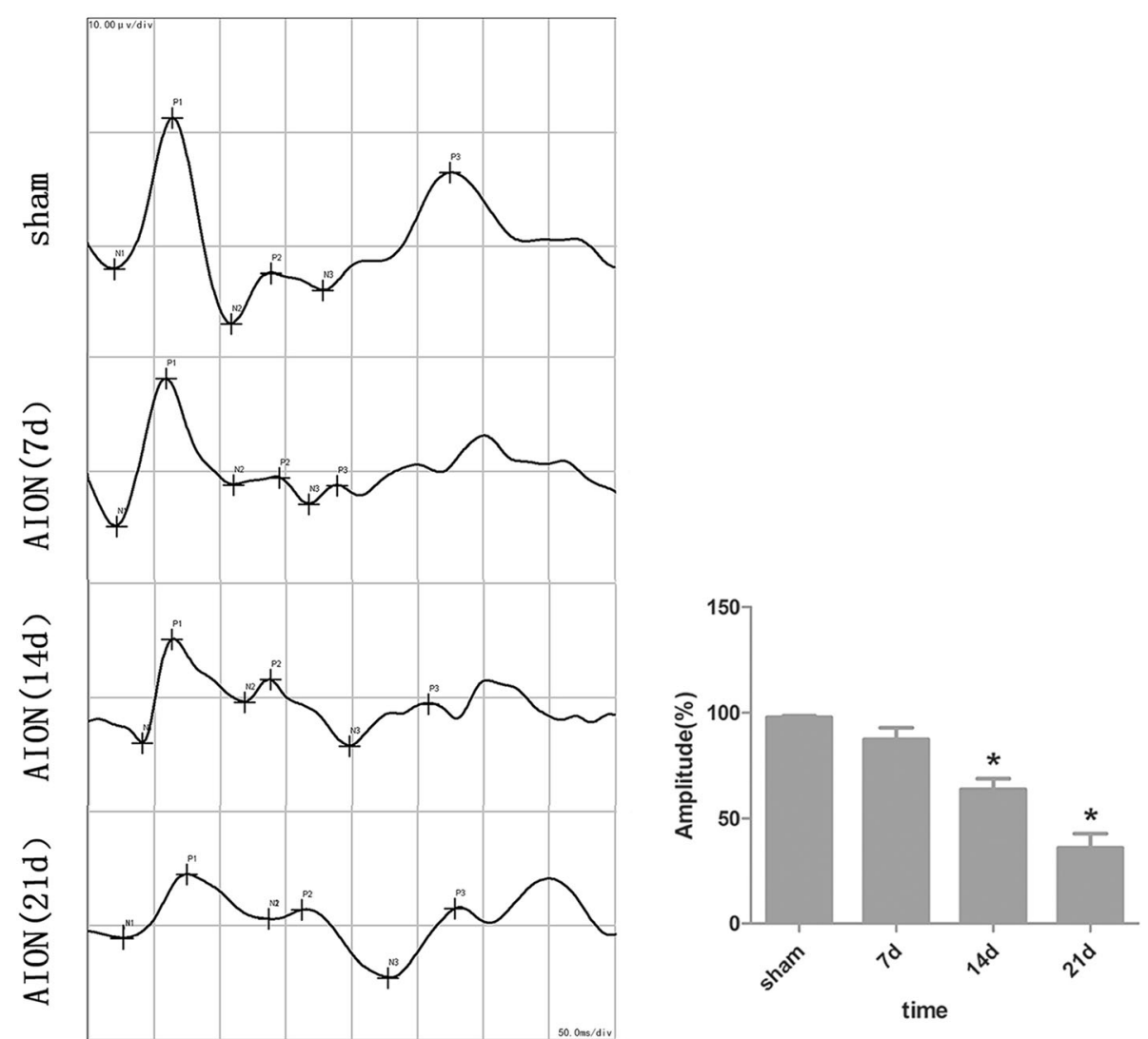

Fig. 2 Flash-Visual Evoked Potentials (F-VEPS) in AION. F-VEP amplitudes of P1 in treated eyes measured $87.3 \pm 11 \%$ of the fellow control eyes at 1 week, $67.6 \pm 11.5 \%$ at 2 week, and $35.9 \pm 13.6 \%$ at 3 week. We did not compare F-VEP latencies in the two data sets because of the indistinctive statistics. ( ${ }^{*} P<0.05$ in the $14 \mathrm{~d}$ and $21 \mathrm{~d}$ groups compared with the sham group, $N=6$ in each group)

of the photosensitizer and the high energy emission of the laser beam. Considering the fact that the intraperitoneal injection with verteporfin has not been previously reported in rodents [20-22], the present study indicates that the slow accumulation of verteporfin in the retinal circulation may have resulted by the slow peritoneal absorption. Furthermore, the intraperitoneal injection of verteporfin is impractical due to the inability to test the peak plasma concentration and the difficulty of mastering the illumination time. Consequently, the mode of delivery was changed from intraperitoneal injection to intravenous injection. Angiography was carried out with verteporfin at a dose of $6 \mathrm{mg} / \mathrm{m}^{2}$ as demonstrated by a previous study [10], in order to test the time duration of the flow of this compound through the choroidal and retinal circulation. Verteporfin was readily detected and was immediately visible in the vascular circulation following the intravenous injection. Following $5 \mathrm{~min}$ of injection, verteporfin was visible in both the choroidal and retinal circulation, and after that time period verteporfin appeared to wash out of the vascular circulation. The detection of verteporfin was not possible in the vascular circulation following
$10 \mathrm{~min}$ of injection. Hence, the operating time was estimated to $10 \mathrm{~min}$ post-injection. This time period was sufficient to complete the laser operation and construct an ideal model for AION.

SD-OCT and FFA images of the treated group indicated significant optic nerve edema compared with the sham group on day 1 following the induction of injury. The development of the edema peaked on day 3 and began to decline from day 7. Based on the SD-OCT images, it was noted that certain treated rats had subretinal fluid on day 1. A similar finding has been demonstrated previously in human AION cases $[9,23]$ and in experimental AION models [24]. The presence of subretinal fluid may promote the death of retinal cells, indicating that the detection of TUNEL-positive cells was possible outside the ganglion cell layer [9]. A wide, non-perfused choroidal vasculature was noted in a certain rat. This unexpected injury may have resulted from individual differences and/or inadvertent operation, and this rat was removed from the study.

TUNEL staining was used to evaluate the apoptotic cell death. Certain parts of TUNEL-positive cells were detected in the outer nuclear layer (ONL) and inner 


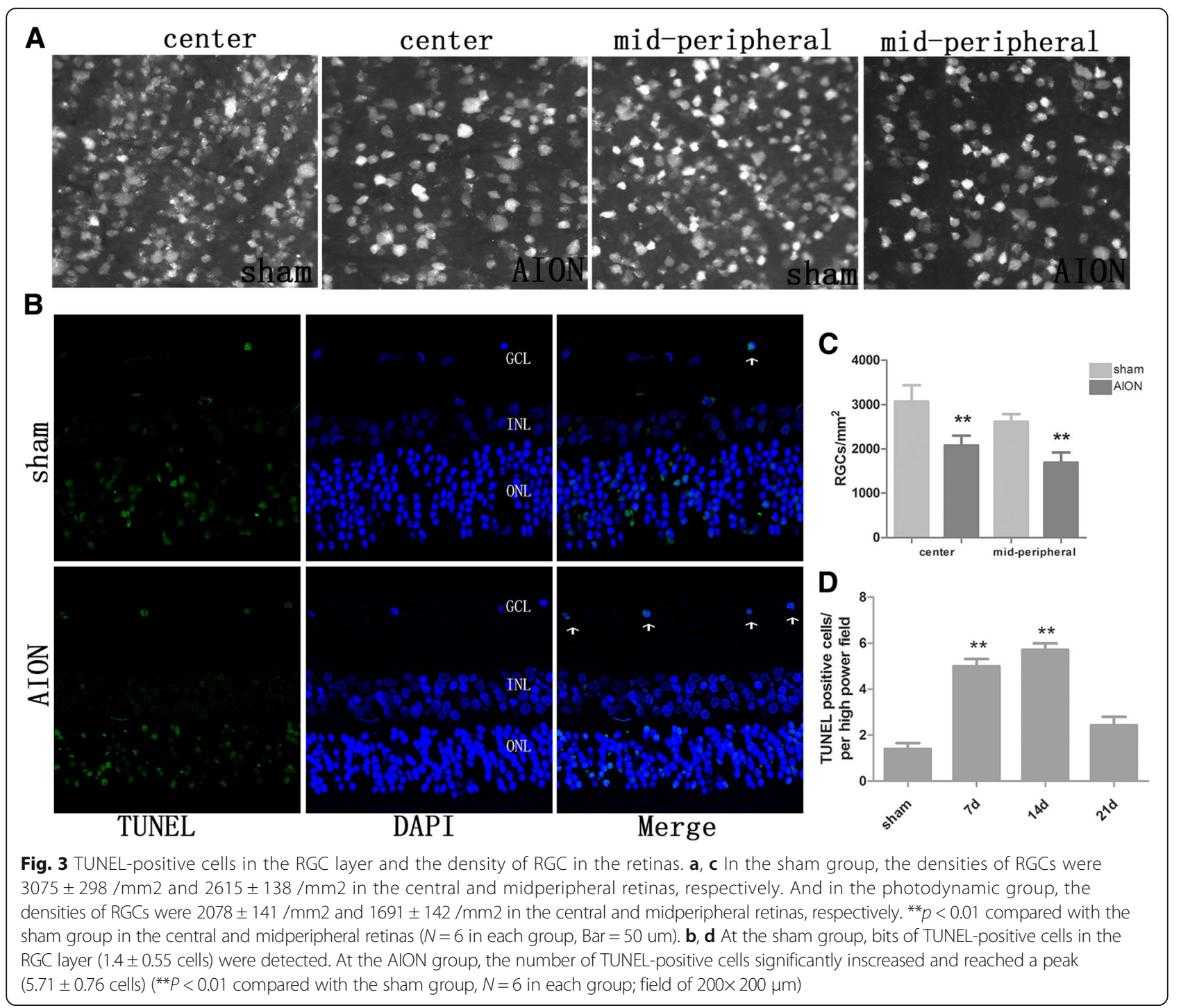

nuclear layer (INL) both in the treated and control eyes, although there was no statistically significant difference $(P=0.3)$ between the two groups. In the treated group, TUNEL-positive cells were demonstrated in the ganglion cell layer by day 7 . The percentage of positive cells peaked by day 14 post-ischemia and subsequently declined, which suggested delayed apoptosis following the $\mathrm{ON}$ infarct compared to the previous studies $[25,26]$. This diversity may have resulted from individual variation and different modeling methods. In the current model, at 3 weeks post-infarct, we could still detect approximately $60 \%$ of all RGCs through retrograde labeling of RGCs with FluoroGold. This time period coincided with the seventh day following the peak of apoptosis. Slater et al. [27] demonstrated that at 2-3 weeks post-ischemia, approximately $50 \%$ of RGCs were still present. As a result, we speculated that the number of RGCs may be reduced considerably after the 3rd week of treatment until the RGC densities were approximately $30 \%$ [8]. The aforementioned findings demonstrated that a prolonged "treatment window" is potentially present in the current model, while the induction of treatment within 7 days post- ischemia may preserve the RGC number. Similarly, such a treatment window may exist in the human AION.

Following injury, extrinsic macrophages were recruited, and resident microglia were activated, at the core of the ischemic ON $[8,28]$. This suggests the breakdown of the blood-ON barrier. Activated macrophages exert a dual fuction: They can enhance remyelination and regeneration [29], while they may produce harmful substances -such as pro-inflammatory mediators which can aggravate the neruronal injury. Due to the individual variation and different modeling methods, the current model exhibited a delay of inflammatory response compared to 


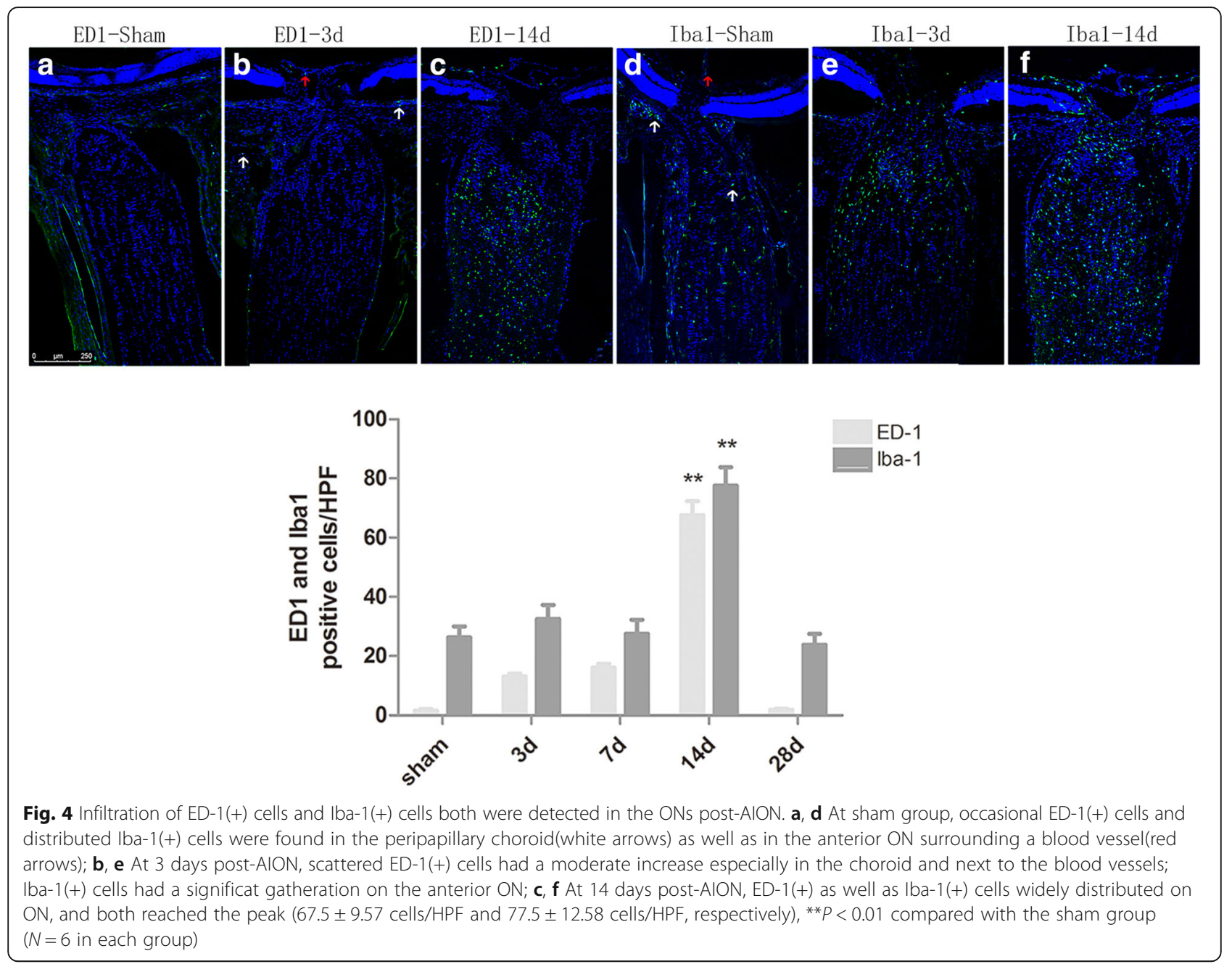

previous studies $[5,28,30]$. Both numbers of $\mathrm{ED}(+)$ and Iba-1(+) cells peaked on the 14th day post-infarct and they were subsequently decreased. This suggests additional time for the modulation of the inflammatory process at an early stage.

The functional deficits were demonstrated by gradually decreasing the amplitude of P1 on F-VEP. According to our histological and pathological findings, the decline of vision resulted from the activation of macrophages and the loss of RGCs. We detected subnormal P1 amplitudes on the 7th day. These observations were more profound on the 14th day post-treatment and suggested that cellular degeneration proceeded following the resolution of the ON edema. The visual function was affected by the progressive damage, which is consistent with the findings reported in previous studies [9, 31, 32].

\section{Conclusion}

An AION model was successfully produced as demonstrated by FFA, SD-OCT and F-VEP images. The model can provide the estimation of inflammation by immunofluorescent staining and the estimation of the number of RGCs. The current study provided a steady, technically simple and controlled model, which can be used to examine the potential neuroprotective effects of certain agents and explore the pathological mechanism of AION.

\section{Abbreviations}

AlON: Anterior ischemic optic neuropathy; CNV: Choroidal neovascularization; DAPI: 4',6-diamidino-2-phenylindole; FFA: Fundus Fluorescein angiography; F-VEP: Flash-visual evoked potential; ON: Optic nerve; ONH: Ptic nerve head; PCV: Polypoidal choroidal vasculopathy; RGCs: Retinal ganglion cells; SD-OCT: Spectral domain optical coherence tomography

\section{Acknowledgements}

Not applicable

\section{Funding}

This study was supported by National Natural Science Foundation of China (no. 81300774), the opening project of Shanghai key laboratory of Fundus Diseases (no.07Z22911)

\section{Availability of data and materials}

All the summarized data is presented in paper. The raw data of the present research is available upon reasonable request from the corresponding author. 


\section{Authors' contributions}

MJY and GYY were primarily responsible for experimental concept and design, and was major contributors in writing the manuscript. LYN raised al the experimental rats and helped to operated on them. ML performed the histological examination of the retina and optic nerve. GQ and WF performed data acquisition and analysis. All authors reviewed and approved the final manuscript.

\section{Ethics approval}

All animal experiments adhered to the ARVO statement for the Use of Animals in Ophthalmic and Vision Research. Ethical approval for this investigation was obtained from the Research Ethics Committee, Shanghai jiaotong University School of Medicine.

\section{Consent for publication}

Not applicable

\section{Competing interests}

The authors declare that they have no competing interests.

\section{Publisher's Note}

Springer Nature remains neutral with regard to jurisdictional claims in published maps and institutional affiliations.

\section{Author details}

'Department of Ophthalmology, Shanghai General Hospital, Shanghai Jiao Tong University School of Medicine, NO.100, Haining Road, Hongkou District, Shanghai 200080, China. ${ }^{2}$ Shanghai Key Laboratory of Ocular Fundus Diseases, NO.100, Haining Road, Hongkou District, Shanghai 200080, China. ${ }^{3}$ Shanghai Engineering Center for Visual Science and Photomedicine, NO.100, Haining Road, Hongkou District, Shanghai 200080, China.

Received: 17 December 2017 Accepted: 12 October 2018 Published online: 22 November 2018

\section{References}

1. Biousse V, Newman NJ. Ischemic optic neuropathies. N Engl J Med. 2015; 372:2428-36. https://doi.org/10.1056/NEJMra1413352.

2. $\mathrm{Xu} \mathrm{L}$, Wang $Y$, Jonas JB. Incidence of nonarteritic anterior ischemic optic neuropathy in adult Chinese: the Beijing eye study. Eur J Ophthalmol. 2007; 17:459-60.

3. Hayreh SS. Management of ischemic optic neuropathies. Indian Ophthalmol. 2011;59:123-36. https://doi.org/10.4103/0301-4738.77024.

4. Bernstein SL, Guo Y, Kelman SE, Flower RW, Johnson MA. Functional and cellular responses in a novel rodent model of anterior ischemic optic neuropathy. Invest Ophthalmol Vis Sci. 2003:44:4153-62.

5. Wen YT, Huang TL, Huang SP, Chang CH, Tsai RK. Early applications of granulocyte colony-stimulating factor (G-CSF) can stabilize the blood-opticnerve barrier and ameliorate inflammation in a rat model of anterior ischemic optic neuropathy (rAION). Dis Model Mech. 2016:9:1193-202. https://doi.org/10.1242/dmm.025999.

6. Huang TL, Wen YT, Chang CH, Chang CW, Lin KH, Tsai RK. Efficacy of intravitreal injections of triamcinolone Acetonide in a rodent model of Nonarteritic anterior ischemic optic neuropathy. Invest Ophthalmol Vis Sci. 2016:57:1878-84. https://doi.org/10.1167/iovs.15-19023.

7. Mathews MK, Guo Y, Langenberg P, Bernstein SL. Ciliary neurotrophic factor (CNTF)-mediated ganglion cell survival in a rodent model of non-arteritic anterior ischaemic optic neuropathy (NAION). Br J Ophthalmol. 2015;99:1337. https://doi.org/10.1136/bjophthalmol-2014-305969.

8. Huang TL, Huang SP, Chang CH, Lin KH, Chang SW, Tsai RK. Protective effects of systemic treatment with methylprednisolone in a rodent model of non-arteritic anterior ischemic optic neuropathy ( $r A I O N)$. Exp Eye Res. 2015;131:69-76. https://doi.org/10.1016/j.exer.2014.12.014.

9. Mantopoulos D, Bouzika P, Tsakris A, Pawlyk BS, Sandberg MA, Miller JW Rizzo lii JF, Vavvas DG, Cestari DM. (TUNEL!!!) an experimental animal model of photodynamic optic nerve head injury (PONHI). Curr Eye Res. 2016:1-9. https://doi.org/10.3109/02713683.2015.1135960.

10. Zacks DN, Ezra E, Terada Y, Michaud N, Connolly E, Gragoudas ES, Miller JW. Verteporfin photodynamic therapy in the rat model of choroidal neovascularization: angiographic and histologic characterization. Invest Ophthalmol Vis Sci. 2002;43:2384-91.
11. Denny CA, Alroy J, Pawlyk BS, Sandberg MA, d'Azzo A, Seyfried TN. Neurochemical, morphological, and neurophysiological abnormalities in retinas of Sandhoff and GM1 gangliosidosis mice. J Neurochem. 2007;101: 1294-302. https://doi.org/10.1111/j.1471-4159.2007.04525.x.

12. Jiang B, Zhang $P$, Zhou D, Zhang J, Xu X, Tang L. Intravitreal transplantation of human umbilical cord blood stem cells protects rats from traumatic optic neuropathy. PLoS One. 2013;8:e69938. https://doi. org/10.1371/journal.pone.0069938.

13. Xiong $S, X u Y$, Ma M, Wang H, Wei F, Gu Q, Xu X. Neuroprotective effects of a novel peptide, FK18, under oxygen-glucose deprivation in SH-SY5Y cells and retinal ischemia in rats via the Akt pathway. Neurochem Int. 2017. https://doi.org/10.1016/j.neuint.2017.02.015.

14. Chen YJ, Huang YS, Chen JT, Chen YH, Tai MC, Chen CL, Liang CM. Protective effects of glucosamine on oxidative-stress and ischemia/reperfusioninduced retinal injury. Invest Ophthalmol Vis Sci. 2015:56:1506-16. https:/doi. org/10.1167/iovs.14-15726.

15. Touitou V, Johnson MA, Guo Y, Miller NR, Bernstein SL. Sustained neuroprotection from a single intravitreal injection of PGJ2 in a rodent model of anterior ischemic optic neuropathy. Invest Ophthalmol Vis Sci. 2013;54:7402-9. https://doi.org/10.1167/iovs.13-12055.

16. Huang SP, Tsai RK. Efficacy of granulocyte-colony stimulating factor treatment in a rat model of anterior ischemic optic neuropathy. Neural Regen Res. 2014;9:1502-5. https://doi.org/10.4103/1673-5374.139472.

17. Fard MA, Ebrahimi KB, Miller NR. RhoA activity and post-ischemic inflammation in an experimental model of adult rodent anterior ischemic optic neuropathy. Brain Res. 2013;1534:76-86. https://doi.org/10.1016/j. brainres.2013.07.053.

18. Bernstein SL, Guo Y. Changes in cholinergic amacrine cells after rodent anterior ischemic optic neuropathy (rAION). Invest Ophthalmol Vis Sci. 2011; 52:904-10. https://doi.org/10.1167/iovs.10-5247.

19. Klaassen CD. Pharmacokinetics of rose bengal in the rat, rabbit, dog and Guinea pig. Toxicol Appl Pharmacol. 1976;38:85-100.

20. She H, Nakazawa T, Matsubara A, Connolly E, Hisatomi T, Noda K, Kim I, Gragoudas ES, Miller JW. Photoreceptor protection after photodynamic therapy using dexamethasone in a rat model of choroidal neovascularization. Invest Ophthalmol Vis Sci. 2008:49:5008-14. https://doi.org/10.1167/iovs.07-1154.

21. Renno RZ, Terada Y, Haddadin MJ, Michaud NA, Gragoudas ES, Miller JW. Selective photodynamic therapy by targeted verteporfin delivery to experimental choroidal neovascularization mediated by a homing peptide to vascular endothelial growth factor receptor-2. Arch Ophthalmol. 2004; 122:1002-11. https://doi.org/10.1001/archopht.122.7.1002.

22. Matsubara A, Nakazawa T, Noda K, She H, Connolly E, Young TA, Ogura Y, Gragoudas ES, Miller JW. Photodynamic therapy induces caspase-dependent apoptosis in rat CNV model. Invest Ophthalmol Vis Sci. 2007;48:4741-7. https://doi.org/10.1167/iovs.06-1534.

23. Hedges TR 3rd, Vuong LN, Gonzalez-Garcia AO, Mendoza-Santiesteban CE, Amaro-Quierza ML. Subretinal fluid from anterior ischemic optic neuropathy demonstrated by optical coherence tomography. Arch Ophthalmol. 2008; 126:812-5. https://doi.org/10.1001/archopht.126.6.812.

24. Yu C, Ho JK, Liao YJ. Subretinal fluid is common in experimental nonarteritic anterior ischemic optic neuropathy. Eye (Lond). 2014;28:1494-501. https://doi.org/10.1038/eye.2014.220.

25. Zhang C, Guo Y, Slater BJ, Miller NR, Bernstein SL. Axonal degeneration, regeneration and ganglion cell death in a rodent model of anterior ischemic optic neuropathy (rAION). Exp Eye Res. 2010;91:286-92. https://doi. org/10.1016/j.exer.2010.05.021.

26. Slater BJ, Mehrabian Z, Guo Y, Hunter A, Bernstein SL. Rodent anterior ischemic optic neuropathy ( $\mathrm{r} \mathrm{AION}$ ) induces regional retinal ganglion cell apoptosis with a unique temporal pattern. Invest Ophthalmol Vis Sci. 2008; 49:3671-6. https://doi.org/10.1167/iovs.07-0504.

27. Tsai RK, Chang $\mathrm{CH}$, Wang HZ. Neuroprotective effects of recombinant human granulocyte colony-stimulating factor (G-CSF) in neurodegeneration after optic nerve crush in rats. Exp Eye Res. 2008;87:242-50. https://doi.org/ 10.1016/j.exer.2008.06.004.

28. Salgado C, Vilson F, Miller NR, Bernstein SL. Cellular inflammation in nonarteritic anterior ischemic optic neuropathy and its primate model. Arch Ophthalmol. 2011;129:1583-91. https://doi.org/10.1001/archophthalmol.2011.351.

29. Yin $Y$, Henzl MT, Lorber B, Nakazawa T, Thomas $T$, Jiang F, Langer $R$, Benowitz LI. Oncomodulin is a macrophage-derived signal for axon regeneration in retinal ganglion cells. Nat Neurosci. 2006;9:843-52. https:// doi.org/10.1038/nn1701. 
30. Zhang C, Guo Y, Miller NR, Bernstein SL. Optic nerve infarction and postischemic inflammation in the rodent model of anterior ischemic optic neuropathy (rAION). Brain Res. 2009;1264:67-75. https://doi.org/10.1016/j. brainres.2008.12.075.

31. Bernstein SL, Johnson MA, Miller NR. Nonarteritic anterior ischemic optic neuropathy (NAION) and its experimental models. Prog Retin Eye Res. 2011; 30:167-87. https://doi.org/10.1016/j.preteyeres.2011.02.003.

32. Slater BJ, Vilson FL, Guo Y, Weinreich D, Hwang S, Bernstein SL. Optic nerve inflammation and demyelination in a rodent model of nonarteritic anterior ischemic optic neuropathy. Invest Ophthalmol Vis Sci. 2013;54:7952-61. https://doi.org/10.1167/iovs.13-12064.

Ready to submit your research? Choose BMC and benefit from:

- fast, convenient online submission

- thorough peer review by experienced researchers in your field

- rapid publication on acceptance

- support for research data, including large and complex data types

- gold Open Access which fosters wider collaboration and increased citations

- maximum visibility for your research: over $100 \mathrm{M}$ website views per year

At BMC, research is always in progress.

Learn more biomedcentral.com/submissions 